\title{
Laser Micromachining of PEDOT:PSS/Graphene Thin Films by Using Beam Shaping Technology
}

\author{
Chien-Kai Chung, Shih-Feng Tseng, Wen-Tse Hsiao, Donyau Chiang and Wei-Cheng Lin \\ Instrument Technology Research Center, National Applied Research Laboratories, Taiwan, No.20, \\ R\&D Rd. VI, Hsinchu Science Park, Hsinchu, 30076, Taiwan \\ E-mail: ckchung@itrc.narl.org.tw
}

\begin{abstract}
This study investigated the interaction between square top-hat laser beams and PEDOT:PSS [poly(3,4-ethylene dioxythiophene):poly(4-styrene sulfonate)]/graphene thin films by using ultraviolet (UV) laser-beam-shaping technology for electrode patterning. This novel laser process can reduce the number of fabrication steps and quantity of chemical solutions and can improve the removal efficiency of graphene films. The laser processing parameters were the laser pulse energy, pulse repetition frequency, and feeding rate of a motorized XY-axis positioning table for ablating the graphene thin films. The square top-hat laser beam was irradiated along line patterns with the applied laser fluences markedly over the ablation thresholds of 1.41 to $2.5 \mathrm{~J} / \mathrm{cm}^{2}$. Increasing the laser fluence from 1.41 to $2.5 \mathrm{~J} / \mathrm{cm}^{2}$ increased the ablated line widths and depths from $35.61 \pm 0.28$ to 43.23 $\pm 0.21 \mu \mathrm{m}$ and from $224 \pm 10$ to $279 \pm 9.29 \mathrm{~nm}$, respectively. The ablated lines of the microstructured electrodes had a clear, smooth, and regular ablated edge quality. Moreover, a maskless laser direct writing process yielded patterned film structures that provide electrical isolation and prevent electrical contact from each region.
\end{abstract}

DOI: $10.2961 /$ jlmn.2016.03.0020

Keywords: ultraviolet laser, PEDOT:PSS/graphene thin film, beam shaping technology, line pattern, surface morphology.

\section{Introduction}

Graphene has attracted considerable attention because it meets the requirements of high transparency and conductivity. Because graphene has high transmittance of more than $90 \%$ and high carrier mobility of up to $2 \times 105 \mathrm{~cm}^{2} / \mathrm{Vs}$, this transparent conductive material is now more efficient than other transparent conductive oxides. Graphene ink applied to glass substrates, because of its lower thickness and high hardness, is an acceptable conductive material for flat panel displays, touch panels, flexible electronics, dyesensitized solar cells, light-emitting diodes, and other optoelectronics products [1-5]. Furthermore, the traditional electrode patterning of thin films involves photolithography processes. However, the process entails complex steps and requires an environment containing harmful chemicals, and photolithography equipment is expensive. Semaltianos et al. [6] used a picosecond pulsed laser with a wavelength of $1064 \mathrm{~nm}$, pulse width of $10.4 \mathrm{ps}$, and pulse repetition rate of $50 \mathrm{kHz}$ to pattern PEDOT:PSS [poly(3,4-ethylene dioxythiophene):poly(4-styrene sulfonate)] thin films coated on glass substrates. After the single pulse ablation tests, the values of ablated threshold were measured equal to 0.13 to $0.18 \mathrm{~J} / \mathrm{cm}^{2}$ for PEDOT:PSS thin films with thicknesses ranging from 100 to $600 \mathrm{~nm}$. The experimental observations revealed that the PEDOT:PSS thin films were ablated by photomechanical ablation. Tseng et al. [7] proposed a nanosecond pulsed ultraviolet (UV) laser system to pattern an electrode layer for isolation on PEDOT:PSS thin films. After the single pulse laser irradiation, the ablation threshold of the PEDOT:PSS film with a thickness of $330 \mathrm{~nm}$ was $0.135 \pm 0.003 \mathrm{~J} / \mathrm{cm}^{2}$. When the laser fluences were increased from 0.566 to $3.11 \mathrm{~J} / \mathrm{cm}^{2}$, the corresponding pat- terned line depths increased from $272 \pm 12 \mathrm{~nm}$ and $507 \pm 4$ $\mathrm{nm}$, respectively. After the line patterning with the laser fluence of $1.7 \mathrm{~J} / \mathrm{cm}^{2}$ and $90 \%$ overlapping rate, the ablated regions had clean, smooth, and straight structures without PEDOT:PSS debris in the patterned line or around neighbouring bank. Chang et al. [8] presented UV laser ablation techniques for screen-printed graphene films coated on glass substrates. Raman spectra of graphene films revealed that the characteristics of the entire multilayer graphene film, and the ratio of the $2 \mathrm{D}$ band to the peak intensity of the $\mathrm{G}$ band was 0.37 . The thickness of multilayer graphene was $2.68 \mu \mathrm{m}$. The low laser fluence of $0.32 \mathrm{~J} / \mathrm{cm}^{2}$ at the scanning speed of $250 \mathrm{~mm} / \mathrm{s}$ produced in less heat accumulation and minimal heat-affected zone (HAZ) on the ablated film surface. Moreover, the ablated line depths were $1.43 \mu \mathrm{m}$ and $2.49 \mu \mathrm{m}$ after laser ablating with laser fluences of $0.32 \mathrm{~J} / \mathrm{cm}^{2}$ and $16.3 \mathrm{~J} / \mathrm{cm}^{2}$, respectively. Sahin et al. [9] adopted a chirped-pulse amplification system with a central wavelength of $1030 \mathrm{~nm}$ and pulse width of 550 fs to ablate single-layer graphene on a $\mathrm{SiO}_{2} / \mathrm{Si}$ substrate. Ablation channels $400 \mathrm{~nm}$ in width were obtained when the laser energy and translation speed of a three-axis piezo stage were $90 \mathrm{~nJ}$ and $330 \mu \mathrm{m} / \mathrm{s}$, respectively. By using the beam shaping technology to change the laser beam from a circular to a rectangular that has more advantages including: (a) the reduce the central energy and damage for ablation processing.(b) To obtain the straight edge along the ablated line and residual stress , and (c) To increase the machining speed.

In the present study, the ablated lines of microstructured electrodes provided electrical isolation and prevented electrical contact from each region through the adoption of a 
maskless laser direct writing process. The laser processing parameters consisted of the laser fluence, pulse repetition frequency, and feeding rate of the XY-axis positioning table used to ablate the thin films. The laser fluence was applied to investigate the machined quality as well as the isolated line width and depth. The surface morphology, threedimensional (3D) topography, and profile of the isolated lines after laser microstructuring were measured using a confocal laser scanning microscope. Moreover, a four-point probe instrument was used to measure the sheet resistance of composite films.

\section{Experimental details}

\subsection{UV laser processing system}

Figure 1 shows the schematic setup of the UV laser processing system. The UV laser (Coherent, Inc. model AVIA 355-14) processing system with a wavelength of 355 $\mathrm{nm}$ is used to ablate isolation lines on PEDOT:PSS/graphene composite thin films used in cell phone touch panels. The laser beam is delivered through three reflective mirrors, a beam expander with $2 \times$ magnification, a beam shaper. The specifications of the UV laser in this system include a maximum power of $16.8 \mathrm{~W}$, transverse mode of TEM $\mathrm{T}_{00}$, pulse repetition rate of $1-300 \mathrm{kHz}$, and pulse width of $28 \mathrm{~ns}$. The square spot size of the full width at half maximum (FWHM) is approximately $70 \times 70 \mu \mathrm{m}$. The output laser power and pulse repetition frequency of pulsed UV lasers and feeding rate of the XY-axis positioning table can be adjusted through a human machine interface for monitoring and controlling the processes.

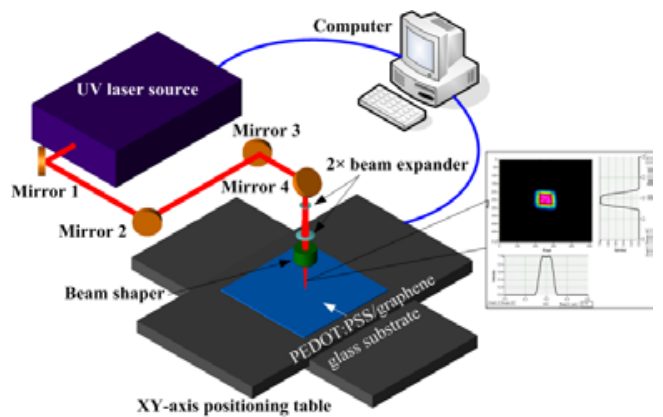

Fig. 1 Schematic setup of the UV laser processing system.

\subsection{Sample preparation}

Through a roller imprinting process, PEDOT:PSS/graphene composite ink was applied to 0.7-mmthick Corning EXG 2000 glass substrates with a thin film thickness of approximately $220 \mathrm{~nm}$. The composite inks were purchased from Legend Star International Co. Ltd. Jiangsu, Taiwan. Figure 2 shows a top view (a) and a crosssectional view (b) of the PEDOT:PSS/graphene film on the glass substrate, which was measured using a dual-beam microscope combined with a focused ion beam and a scanning electron microscope (SEM). Before the SEM observation, a focused ion beam (FIB) system was used to mill a step for easy thickness observation. To protect the specimen surface during the FIB process, the platinum $(\mathrm{Pt})$ was coated on PEDOT:PSS/graphene film surface. In this article, the sample is referred to as a PEDOT:PSS/graphene glass substrate. Figure 3 shows the surface roughness in a 3D image of the PEDOT:PSS/graphene composite film scanned using an atomic force microscope (Veeco di Dimension 3100, USA). The surface measurement area was 1 $\times 1 \mu \mathrm{m}^{2}$. The measured values of the root mean square (Rms) and arithmetical mean deviation ( $\mathrm{Ra}$ ) of the roughness profile were $3.17 \mathrm{~nm}$ and $2.53 \mathrm{~nm}$ for the PEDOT:PSS/graphene film surface, respectively.

Before the laser ablated the isolation lines, a spectrometer (HITACHI U-4001) was used to measure the transmittance and reflectance of the PEDOT:PSS/graphene glass substrate. The measured data at wavelengths ranging from 300 to $800 \mathrm{~nm}$ are shown in Figure 4. The light transmittance and reflectance values of the PEDOT:PSS/graphene glass substrate at a 355-nm wavelength were approximately $77.1 \%$ and $8.6 \%$, respectively. The light absorbance value of this substrate at the UV laser wavelength (355 nm) was approximately $14.3 \%$. Because the substrate has highabsorption characteristics at the 355-nm wavelength, UV laser structuring can improve the removal efficiency of PEDOT:PSS/graphene composite films.

The sheet resistance of the PEDOT:PSS/graphene composite films was approximately $75 \Omega / \square$, which was measured using a four-point probe instrument. The Young's modulus of the PEDOT:PSS/graphene composite films was measured at room temperature $\left(25^{\circ} \mathrm{C}\right)$ by using a nanoindenter (Nanoindenter XP, MTS Systems, Oak Ridge, TN, USA) equipped with a diamond Berkovich tip. To calculate the elastic modulus of the thin films, continuous stiffness measurements were performed to determine the elastic stiffness of the contact according to the slope of the load-displacement data during unloading. Nanoindentation tests conducted at room temperature were performed at six locations for each specimen; the distance between the two nearest locations was approximately $200 \mu \mathrm{m}$. The indenter tip was calibrated immediately before the experiment was conducted by using standard specimens of fused silica $(E=$ $74.0 \mathrm{GPa}$ ). Figure 5 shows the load-displacement curves of composite films coating glass substrates. The measured nanohardness and modulus of the PEDOT:PSS/graphene composite films were $2.45 \pm 0.15$ and $58.1 \pm 2.8 \mathrm{GPa}$, respectively.
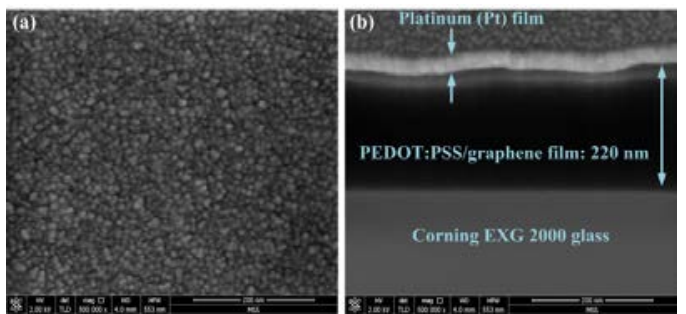

Fig. 2 SEM images of top view (a) and cross-sectional view (b) of the PEDOT:PSS/graphene glass substrate.

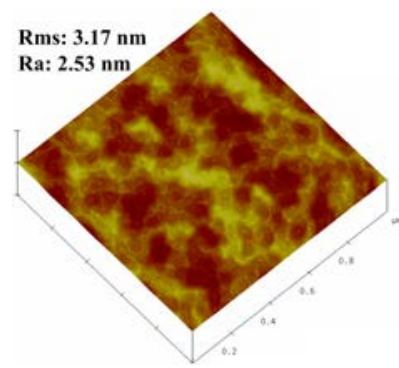

Fig. 3 Surface roughness of the PEDOT:PSS/graphene composite film. 


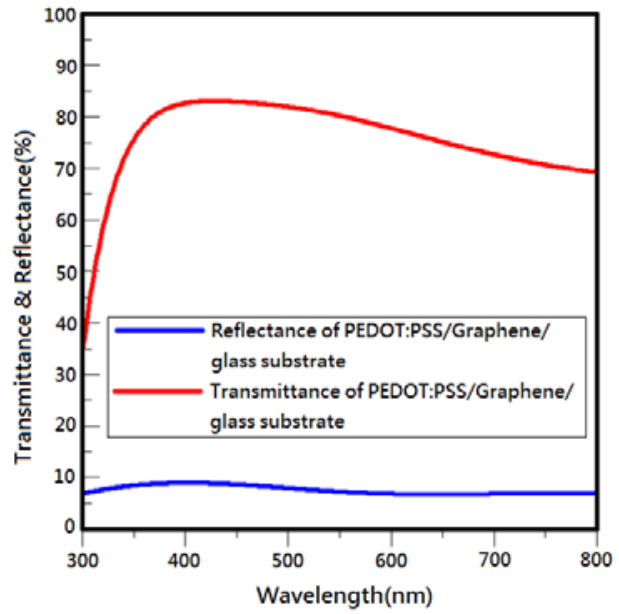

Fig. 4 Light transmittance and reflectance versus wavelength for the PEDOT:PSS/graphene glass substrate.

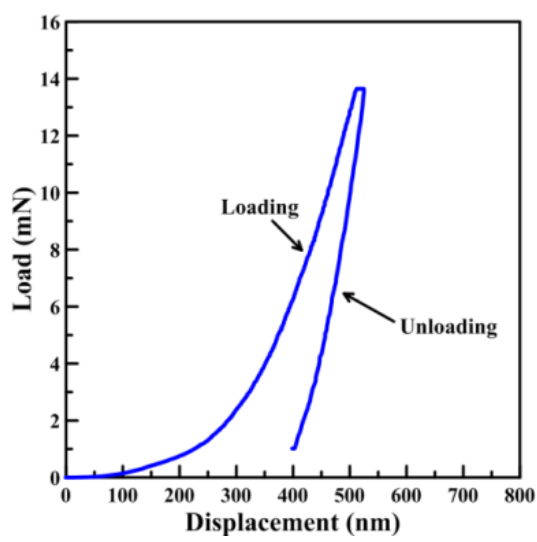

Fig. 5 Load-displacement curves of PEDOT:PSS/graphene composite films coating glass substrates.

\subsection{Top-hat profile generation for single-mode lasers}

Many laser sources (e.g., several solid state lasers, fiber lasers, and gas lasers) operate in a single transverse mode with a well-defined Gaussian beam profile. To obtain a homogeneous top-hat profile from such laser sources, phase-shifting optical elements can be utilized to transform the Gaussian beam profile into a top-hat one. The surface of such an acylindrical lens (aspherical shape in one direction) is described by the following equation [10]:

$$
Z(Y)=\frac{C \cdot Y^{2}}{1+\sqrt{1-(1+K) C^{2} Y^{2}}}+A_{4} Y^{4}+A_{6} Y^{6}+\ldots+A_{n} Y^{n}
$$

where $Z$ is the sag height of a line drawn from a point on the aspherical surface at a radial distance, $C$ is the curvature of the aspherical surface near the optical axis, $Y$ is the radial distance from the optical axis, $K$ is the conic constant, and $A_{4}, A_{6}$, and $A_{n}$ are the high order aspheric coefficient.

The principle of beam shaping with a free-form phaseshifting element is shown in Figure 6 for one direction, exemplarily. A collimated Gaussian beam with a welldefined beam diameter and divergence impinges on a refractive free-form aspherical optical element. The surface is designed so that a redistribution of the intensity profile from Gaussian to top hat is achieved through spatial phase shifting. To obtain a beam with a uniform intensity distribution, a beam shaper (RONAR-SMITH®: BS-355) optical component was used. A UV laser source was passed through a beam expander, beam shaper, and focusing lens to obtain a uniform laser beam with a near top-hat intensity distribution. After beam shaping, the top-hat intensity profile of the laser beam, of which cross sections on the $\mathrm{x}$ - and y-axes are shown in the Figure 7, is often desirable in machining applications such as laser ablation and micropatterning. The square spot size of the FWHM is approximately $70 \mu \mathrm{m} \times 70 \mu \mathrm{m}$.

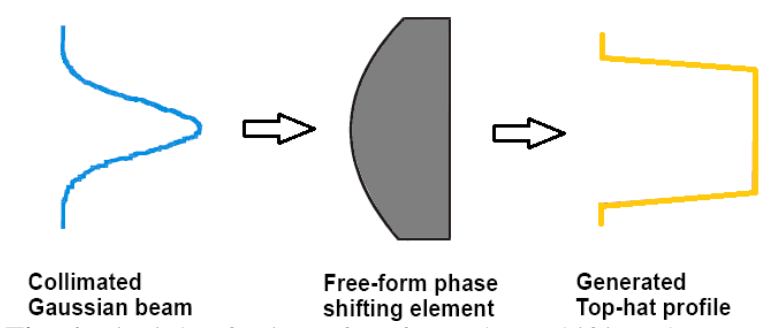

Fig. 6 Principle of using a free-form phase-shifting element to transform the impinging Gaussian beam profile into a top-hat profile.

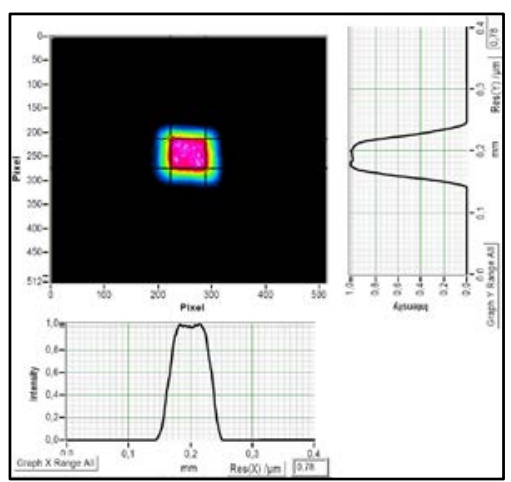

Fig. 7 Measured beam profile generated after beam shaping from a UV laser at $365 \mathrm{~nm}$.

\section{Experimental results}

\subsection{Ablated line width and depth}

To obtain the surface morphology of the ablated lines on PEDOT:PSS/graphene composite films, the focused UV laser beam was irradiated along line patterns with the applied laser fluences markedly higher than the ablation thresholds of $1.41,1.56,1.73,1.87,2.05,2.19,2.36$, and $2.5 \mathrm{~J} / \mathrm{cm}^{2}$. The laser fluence $(F)$ could be calculated by the following equation:

$$
F=\frac{E}{A}
$$

where $E$ and $A$ are the laser pulse energy and the area of laser spots, respectively.

Figure 8 shows the relationship of the ablated line widths and depths with the various laser fluences on the PEDOT:PSS/graphene glass substrates. The average values from five measured points on each specimen with the corresponding standard deviation were introduced. The measured results showed that the ablated line width and depth increased linearly with the laser fluence. The ablated line widths and depths increased from $35.61 \pm 0.28$ to $43.23 \pm 0.21 \mu \mathrm{m}$ and from $224 \pm 10$ to $279 \pm 9.29 \mathrm{~nm}$, respectively, as the laser fluence was increased from 1.41 to $2.5 \mathrm{~J} / \mathrm{cm}^{2}$.The increase in laser fluence caused a larger thermal diffusion increase and increased the size of the 
ablated spots [11]. The ablated line depths increased from $270 \pm 4.93$ to $271 \pm 4.58 \mathrm{~nm}$ as the laser fluence was increased from 2.19 to $2.36 \mathrm{~J} / \mathrm{cm}^{2}$. Because the PEDOT:PSS/graphene composite films had a thickness of $220 \mathrm{~nm}$, the fluence intensities within these ranges sufficed for removing the film completely without producing marks on the glass substrate. Increasing the laser fluence to $2.5 \mathrm{~J} / \mathrm{cm}^{2}$ increased the ablated line depth to 279 $\pm 9.29 \mathrm{~nm}$. The powerful fluence intensities produced slightly ablated marks on the glass substrate. Because the laser fluence was less than $2.05 \mathrm{~J} / \mathrm{cm}^{2}$, the PEDOT:PSS/graphene composite film was partially ablated along the ablated line path, and the electrode structure could not provide electrical isolation and prevent electrical contact from each region.

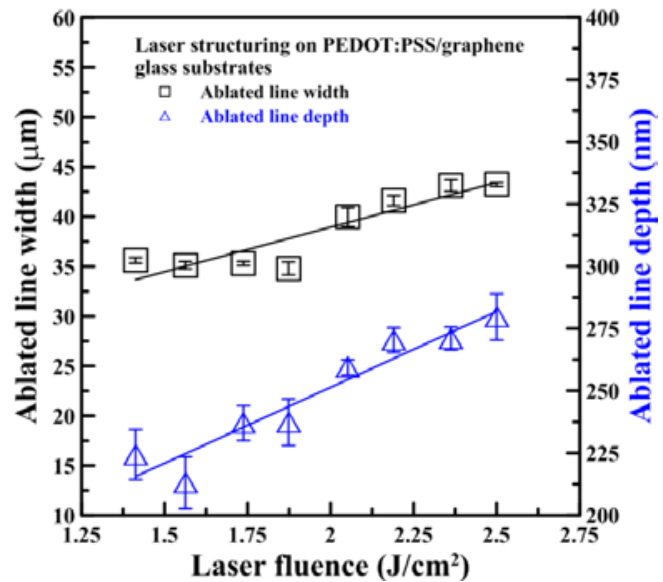

Fig. 8 Relationships of the ablated line widths and depths at various laser fluences on the PEDOT:PSS/graphene glass substrates.

\subsection{Surface profiles of the ablated line}

Overlapping rate of laser focusing spot was an important factor for laser processing. Moreover, removal rate of thin films was affected by the overlapping rate. The values of the feeding rate $(V)$ of the motorized $X Y$-axis positioning table and the laser pulse repetition frequency $\left(P_{R F}\right)$ were used to calculate the overlapping rate $\left(O_{R}\right)$ of square spots and to determine the patterning quality. The $O_{R}$ value of laser spots could be defined and estimated as follows:

$$
O_{R}=\left(1-\frac{V}{P_{R F} \times l}\right) \times 100 \%
$$

where $l$ is the side length of square laser spots. Throughout this experimental work, the spot size of the square laser beam at the composite film was fixed at about $70 \mu \mathrm{m} \times 70$ $\mu \mathrm{m}$. The designed ablation line patterns with about $43 \% O_{R}$ were created with a laser pulse repetition frequency of 100 $\mathrm{kHz}$ and a $4000 \mathrm{~mm} / \mathrm{s}$ feeding rate of the motorized $\mathrm{XY}$ axis positioning table.

Figure 9 shows the typical surface morphology, 3D topography, and cross-sectional profile of an ablated line with the fluence of $2.19 \mathrm{~J} / \mathrm{cm}^{2}$ on the PEDOT:PSS/graphene glass substrate, which was measured using the confocal laser scanning microscope. The ablated profile was subjected to a UV laser beam with an average laser power of $3.5 \mathrm{~W}$, pulse repetition frequency of 100 $\mathrm{kHz}$, and feeding rate of the $\mathrm{XY}$-axis positioning table of $4000 \mathrm{~mm} / \mathrm{s}$. The measured results revealed that the PEDOT:PSS/grapheme composite films were uniformly re- moved along the ablated line path. The laser microstructuring process left a clean ablated surface with no film debris in the ablated line or around the neighbouring film surface. The laser-ablated line had regular fish-scale shapes (laser ablated marks) on the ablated edge and no heat-affected zone near the ablated edges. However, the ablated film was curled, resulting in pileup around the line edge, which formed a shoulder with sharp edges, as shown in the crosssectional profile. The height of the shoulder from the film surface to the peak-curled edge was up to several hundred nanometers. Figure 10 shows the typical surface morphology, 3D topography, and cross-sectional profile of an ablated line with the fluence of $1.41 \mathrm{~J} / \mathrm{cm}^{2}$ on the PEDOT:PSS/graphene glass substrate. The ablated profile was subjected to a UV laser beam with an average laser power of $2.26 \mathrm{~W}$, pulse repetition frequency of $100 \mathrm{kHz}$, and feeding rate of the XY-axis positioning table of $4000 \mathrm{~mm} / \mathrm{s}$. The measured results regarding the surface morphology revealed that the residual PEDOT:PSS/graphene film was produced on the ablated line path with a laser fluence of less than $2.19 \mathrm{~J} / \mathrm{cm}^{2}$. Comparing the cross-sectional profiles of the ablated lines revealed that the ablated line width in Figure 9 is obviously larger than that in Figure 10, because of the high laser fluence. Moreover, the ablated line clearly had a curled edge and nanoscale shoulder. Because the PEDOT:PSS/graphene composite films were ablated by photomechanical ablation, the laser ablation with the lower fluence produced high shoulder near the ablated line edge [6].
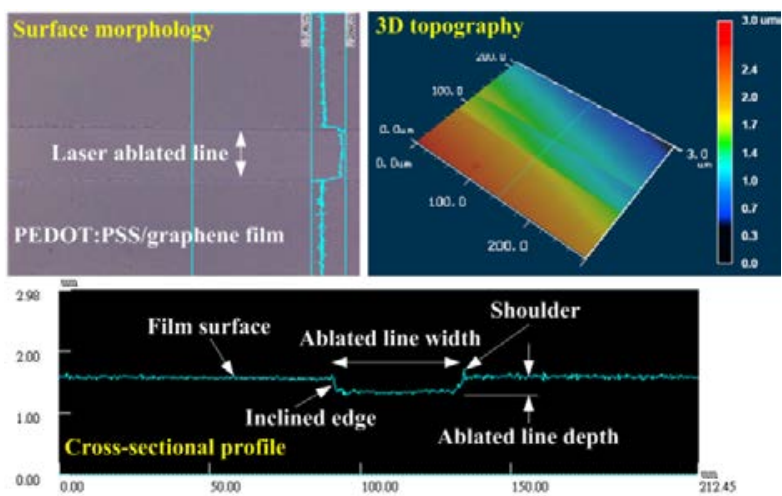

Fig. 9 Typical surface morphology, 3D topography, and crosssectional profile of the ablated line with a laser fluence of 2.19 $\mathrm{J} / \mathrm{cm}^{2}$ on the PEDOT:PSS/graphene glass substrate.
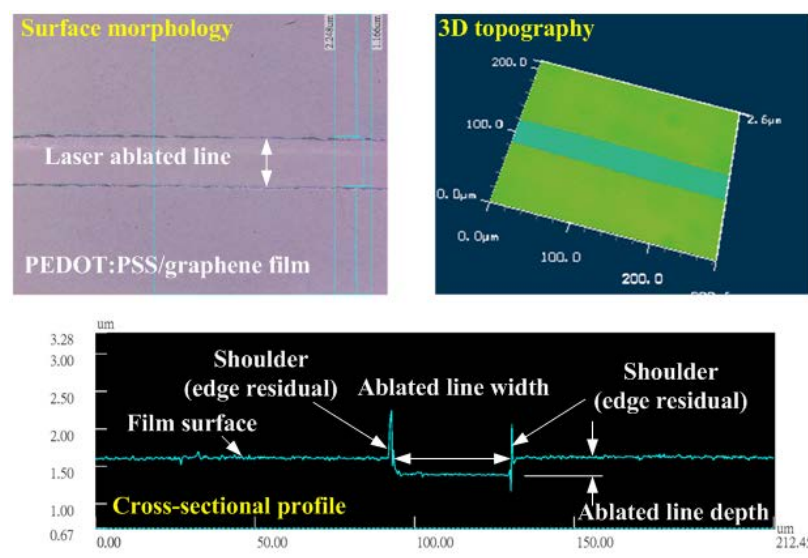

Fig. 10 Typical surface morphology, 3D topography, and crosssectional profile of the ablated line with a laser fluence of 1.41 $\mathrm{J} / \mathrm{cm}^{2}$ on the PEDOT:PSS/graphene glass substrate. 


\section{Conclusions}

This study successfully used high-speed direct writing technology with a nanosecond pulsed UV laser and UV laser-beam-shaping technology to selectively remove PEDOT:PSS/graphene composite films for isolated line patterning. The laser ablated lines had nanoscale shoulders, sharp edges, smooth plane, and straight lines. The glass substrate was undamaged when the laser fluence was set between 2.19 and $2.36 \mathrm{~J} / \mathrm{cm}^{2}$. Moreover, the ablated line widths and depths increased from $41.61 \pm 0.5$ to $43.13 \pm$ $0.61 \mu \mathrm{m}$ and from $270 \pm 4.93$ to $271 \pm 4.58 \mathrm{~nm}$, respectively. The ablated line width and depth values increased with the laser fluence. The curled films around the ablated line edge formed a shoulder, the height of which varied with the fluence. After laser microstructuring of the isolated patterns, clear and completely isolated lines were achieved by setting the laser fluence to $2.19 \mathrm{~J} / \mathrm{cm}^{2}$ and using UV laserbeam-shaping technology on the PEDOT:PSS/graphene glass substrate.

\section{Acknowledgment}

The authors thank the Ministry of Science and Technology of Taiwan for financially supporting this research under projects MOST 105-2221-E-492-015 and MOST 105-2622-E-492-011-CC3.

\section{References}

[1] J. Wu, H.A. Becerril, Z. Bao, Z. Liu, Y. Chen, P. Peumans: Appl. Phys. Lett., 92, (2008) 263302.

[2] G. Jo, M. Choe, C.Y. Cho, J.H. Kim, W. Park, S. Lee, W.K. Hong, T.W. Kim, S.J. Park, B.H. Hong, Y.H. Kahng, T. Lee: Nanotechnology, 21, (2010) 175201.

[3] X. Wang, L. Zhi, K. Müllen, Transparent: Nano Lett., 8, (2008) 323.

[4] T.L. Chang, Z.C. Chen, W.Y. Chen, H.C. Han, S.F. Tseng: Microelectron. Eng., 158, (2016) 1.

[5] S.F. Tseng, W.T. Haiso, P.Y. Cheng, C.K. Chung, Y.S. Lin, S.C. Chien, W.Y. Huang: Sens. Actuators, B, 226, (2016) 342.

[6] N.G. Semaltianos, C. Koidis, C. Pitsalidis, P. Karagiannidis, S. Logothetids, W. Perrie, D. Liu, S.P. Edwardson, E. Fearon, R.J. Potter, G. Dearden, K.G. Watkins: Synth. Met., 161, (2011) 431.

[7] S.F. Tseng, W.T. Hsiao, C.K. Chung, D. Chiang: Appl. Phys. A, 112, (2013) 41.

[8] T.L. Chang, Z.C. Chen, S.F. Tseng: Appl. Surf. Sci., 374, (2016) 305.

[9] R. Sahin, E. Simsek, S. Akturk: Appl. Phys. Lett., 104, (2014) 053118.

[10]O. Homburg, F. Völkermeyer, F. Toennissen: Conference on Lasers in Manufacturing, (2007) 757.

[11] J. Hermann, M. Benfarah, S. Bruneau, E. Axente, G. Coustillier, T. Itina, J.F. Guillemoles, P. Alloncle: J. Phys. D: Appl. Phys., 39, (2006) 453.

(Received: July 16, 2016, Accepted: October 12, 2016) 